\title{
En búsqueda de la estructura ontológica del derecho**
}

\section{In search of the structural ontology of law}

SUMARIO

1. El reto de la existencia de una estructura ontológica del derecho. 2. Una comprensión pre-reflexiva del derecho. 3. Condiciones necesarias y suficientes. 4. Equilibrio reflexivo. Bibliografía.

\section{RESUMEN}

La tarea central de la teoría del derecho es la de explicar la ontología del derecho o, como comúnmente se señala, su naturaleza. El objetivo de este ensayo es describir de manera general una metodología que permita llevar

* Profesor de Filosofía del Derecho y Derecho Constitucional de la Universidad Externado de Colombia y, durante el curso académico 2010-2011, Senior Research for Scholar de la Yale Law School. Abogado por la Universidad Externado de Colombia (1996), doctor por la Universidad de Salamanca (2001), máster en Humanidades (con énfasis en Filosofía) por la Universidad de la Florida y candidato a doctor en Filosofía por la misma Universidad. Ha impartido conferencias en trece países en Europa, Norteamérica, América Latina y Oceanía. Es autor de más de treinta y cinco artículos científicos y de cinco volúmenes monográficos en filosofía del derecho, derecho constitucional y derecho de la responsabilidad civil extracontractual. Su actividad científica ha sido auspiciada por becas de la Universidad Externado de Colombia, la Agencia Española de Cooperación Internacional (AECI), el Servicio Alemán de Intercambio Académico (DAAD) y la Comisión Fulbright. Contacto: carlos.bernal@uexternado.edu.co El autor agradece a Kirk Ludwig, Robert D'Amico, John Byro, Michael Siebecker, David Copp, Brian Bix y Kevin WaLton por sus comentarios a los primeros borradores de este trabajo presentados en el Instituto de Filosofía del Derecho "Julius Stone" de la Escuela de Derecho de Sydney en abril de 2011 y en el Departamento de Filosofía de la Universidad de Florida en junio de 2011 Asimismo, agradece al traductor por esta versión en castellano del texto original en inglés.

** El artículo original ha sido publicado en Analisi e diritto. Ricerche di giurisprudenza analitica, 2012. Traducción a cargo de GonZalo ViLla Rosas, abogado de la Universidad Externado de Colombia, con estudios en Filosofía en la Universidad Nacional de Colombia; becario del Deutscher Akademischer Austauschdienst (DAAD) y de la Universidad Externado de Colombia (ALECOL) con maestría en Derecho (LL.M.) S.C.L. por la Universidad de Christian-Albrecht de Kiel (Alemania), doctorando en Filosofía del Derecho en la misma universidad; contacto: gonzalo.villa@uexternado.edu.co 
a cabo investigaciones acerca de la naturaleza del derecho. Se sostiene que investigaciones de este tipo deben empezar con una comprensión pre-reflexiva del derecho en tanto entidad basada en una práctica social. Asimismo, que dichas investigaciones deben tratar de reducir la explicación acerca de la naturaleza del derecho a un catálogo de condiciones necesarias y suficientes para la existencia del derecho, y que el éxito en esta empresa no depende solo del análisis conceptual, sino que precisa también de una construcción teórica que tenga por objeto el logro de un equilibrio reflexivo.

PALABRAS CLAVE

Metodología de la teoría del derecho, análisis conceptual, equilibrio reflexivo, ontología jurídica, naturaleza del derecho.

\section{ABSTRACT}

The central task of jurisprudence is explaining the ontology of law or, as it is commonly said, its nature. The aim of this paper is to outline a methodology for carrying out inquiries into the nature of law. I argue that enquiries of this kind ought to begin with a pre-reflective understanding of law as an entity grounded in a social practice, that they should attempt to provide reductive necessary and sufficient conditions for the existence of law, and that success in this enterprise depends on conceptual analysis although it also requires a theory construction aiming at achieving a reflective equilibrium.

\section{KEYWORDS}

Methodology of jurisprudence, conceptual analysis, reflective equilibrium, legal ontology, nature of law.

\section{EL RETO DE LA EXISTENCIA DE UNA ESTRUCTURA ONTOLÓGICA DEL DERECHO}

La tarea central de la teoría del derecho es la de explicar la ontología del derecho, o como se señala comúnmente, su naturaleza. Durante las últimas tres décadas la teoría del derecho ha dirigido su atención hacia su propia metodología. Ha existido un creciente interés por parte de los teóricos del derecho en explorar preguntas tales como: ¿qué es la teoría del derecho?, ¿cuáles son sus objetos y sus propósitos?, y ¿cómo debe llevarse a cabo una investigación de teoría jurídica? El objetivo de estas preguntas es entender el tipo de empresa a la que se dedican los teóricos del derecho, y evaluar tanto la plausibilidad de sus opciones metodológicas y las razones que dichos teóricos tienen para fundamentar la elección que hacen respecto a dichas 
posibilidades, como también la conexión de ellas con los resultados teóricos finales de las investigaciones en teoría jurídica.

La reflexión acerca de la metodología de la teoría del derecho ha experimentado un renacimiento notable a partir del debate entre H.L.A. HART y Ronald Dworkin. Uno de los aspectos centrales de este debate trata acerca de la pregunta de si una teoría general del derecho puede ser descriptiva. En el epílogo de The Concept of Law, HART explica que su objetivo fue el de "proveer una teoría acerca de lo que es el derecho, que fuera, al mismo tiempo, general y descriptiva"1. HART aclara este punto, al señalar que su teoría es descriptiva en el sentido según el cual:

ella es moralmente neutral y no tiene objetivos justificatorios: ella no busca justificar o recomendar, con base en razones morales o de otra índole, las formas y estructuras que aparecen en [esta] explicación del derecho².

DwORKIN cuestiona la posibilidad de una teoría descriptiva del derecho en el texto revisado de su HaRT Lecture, pronunciada en Oxford en 2001. DWORKIN sostiene:

una teoría general acerca de cómo ha de ser identificado el derecho válido, como la propia teoría de HART, no es una descripción neutral de la práctica jurídica, sino una interpretación de ella que aspira no solo a describirla sino a justificarla - a mostrar por qué la práctica es valiosa y cómo debería ser dirigida de manera que se proteja y enriquezca ese valor. Si ello es así, entonces una teoría jurídica descansa sobre juicios y convicciones morales y éticos ${ }^{3}$.

DwORKIN invoca dos argumentos para fundamentar esta afirmación. En primer lugar, afirma que una teoría, tal como la que ofrece HART, que versa sobre la naturaleza del concepto del derecho, trata sobre un concepto político. Teorías acerca de la naturaleza de este tipo de conceptos (tales como los de justicia, libertad, igualdad, democracia y derecho) son necesariamente no descriptivas

1 HART, 1994, 239.

2 HART, 1994, 240. Jules COLEMAn señala que la caracterización de HART de su proyecto como descriptivo solo implica la afirmación según la cual "una teoría de la ciencia jurídica no requiere garantizar la inferencia de la legalidad a la legitimidad moral": véase CoLEMAN, 2002, 312.

3 Dworkin, 2004, 2. Reimpreso en: Dworkin, 2006, 140-186. Este pasaje se encuentra en 140-141. De aquí en adelante citaré este artículo a partir de Dworkin, 2006 (Justice in Robes). Dworkin no sostiene de manera explícita que sea imposible que exista una teoría jurídica descriptiva. No obstante, esta parece ser una interpretación razonable de su réplica a HART. Esta interpretación puede deducirse a partir de la caracterización que hace DwORKIN de las teorías del estilo de aquella que expone HART, como una interpretación (evaluativa) y no como una mera descripción de la práctica jurídica, y a partir de su afirmación conforme a la cual "la ambición de HART de proveer una solución puramente descriptiva al problema central de la filosofía del derecho es errónea": ibíd., 143. 
en el sentido antes señalado ${ }^{4}$. Dworkin piensa que estas teorías son necesariamente interpretativas, es decir, que ellas dependen necesariamente, al menos en parte, de ciertos hechos evaluativos ${ }^{5}$. Explicar la naturaleza del concepto del derecho implica necesariamente valerse de argumentos normativos o prácticos. Defender una explicación de dicha naturaleza "no significa otra cosa que defender una teoría controversial acerca de la moralidad política"6.

El segundo argumento afirma que el término "descriptivo" es ambiguo, y que cada uno de los sentidos en los cuales podemos entender el proyecto de describir el derecho "resulta ser manifiestamente inaplicable" ${ }^{7}$. Tal proyecto, o bien puede consistir en un "análisis semántico" "que tiene como fin descubrir los criterios que la gente común y corriente usa realmente [...] cuando describe algo" como legal o ilegal; o bien puede ser un "proyecto estructural", que "tenga como objetivo descubrir la verdadera esencia de lo que la gente describe de esta forma"; o bien puede ser la búsqueda de una generalización "empírica", "estadística", "histórica", "sociológica" o "antropológica" acerca de lo que la gente considera como derecho ${ }^{8}$.

Por medio de argumentos bien conocidos, como los del desacuerdo teórico y el aguijón semántico, Dworkin se esfuerza por mostrar que el proyecto de análisis semántico no puede prosperar. El objetivo de este proyecto es descubrir algunos criterios comunes que determinan cuándo las proposiciones de derecho son verdaderas -es decir, las proposiciones por medio de las cuales el derecho prohíbe, manda o permite ciertas acciones, o atribuye poderes a alguien- ${ }^{9}$. DwORKIN niega la existencia de tales criterios comunes, los cuales denomina fundamentos del derecho. Este autor sostiene que no existe acuerdo entre los operadores jurídicos acerca de los fundamentos del derecho. Dworkin denomina a este desacuerdo como desacuerdo teórico. Y, parece considerar también los fundamentos del derecho como los criterios que proveen significado a la palabra "derecho" y como los criterios que determinan cuándo el uso de esta palabra es correcto ${ }^{10}$. De esta forma, no existe

4 Dworkin, 2006, 149 (y en 162 acerca del derecho como un concepto político).

5 Acerca de los hechos evaluativos como elementos necesarios de las teorías interpretativas véase STAVRopoulos, 2003.

6 DWORKIN, 2011, 404.

7 DwORKIN, 2006, 147.

8 DWORKIN, 2006, 150.

9 Dworkin, 2006, 151 y 156. Para una explicación de estos argumentos véase SMITH, 2010, 635-661.

10 SмIтн explica la doble función de estos criterios, al sostener que Dworkin trata los criterios contenidos en la regla de uso de la palabra "derecho" como aquellos criterios que exponen además los fundamentos del derecho: véase SмIтн, 2010, 645. Un ejemplo puede ilustrar esta doble función. Supóngase que la justicia es un criterio que provee el significado de "derecho". Así, "derecho" significaría un ente justo con ciertas propiedades adicionales. La expresión: "derecho justo" sería redundante; y la expresión "derecho injusto" sería un oxímoron. En consecuencia, una afirmación de derecho debe ser aceptada si, y solo si, es justa; y una proposición de derecho de acuerdo con la cual "el derecho ordena" sería verdadera si, y solo si, es justo. 
acuerdo acerca de los criterios que determinan el significado de la palabra "derecho"11. En consecuencia, el proyecto de análisis semántico no puede lograr la descripción de la naturaleza del derecho, dado que no hay certeza alguna acerca de lo que se describe. No existe ningún criterio común acerca de los fundamentos de nuestro uso de la palabra "derecho". No existe ningún criterio que sea seguro, de manera tal que pueda revelarnos la naturaleza del derecho. Y aún peor, dado que el proyecto de análisis semántico presupone la existencia de tales criterios comunes, soslaya la explicación de un elemento importante de la naturaleza del derecho: precisamente, la explicación de la existencia de los desacuerdos teóricos ${ }^{12}$.

Con respecto al proyecto de la generalización empírica, DwORKIN sostiene que la teoría de HART no es el resultado de estudio empírico alguno, y que aun si lo fuese, fallaría como explicación de la naturaleza del derecho, porque existen contraejemplos que sería posible oponer a ella. En concreto, existen proposiciones acerca del derecho que son consideradas verdaderas, pero que, sin embargo, no cumplen los criterios de la teoría de HART (los criterios de la tesis de las fuentes o, en otras palabras, la existencia de una regla de reconocimiento aceptada por los funcionarios de la ley que hace que ciertas proposiciones acerca del derecho sean verdaderas) ${ }^{13}$.

Por último Dworkin sostiene también que el proyecto estructural falla. Este proyecto consiste en conocer cierto tipo de hechos que definen la esencia de ciertos objetos y, en consecuencia, en determinar en qué condiciones un concepto se usa de manera correcta al hablar acerca de dicha esencia. Los puntos de vista de Dworkin acerca de este asunto son complejos. Por una parte, este autor piensa que este proyecto, en tanto proyecto descriptivo, solo es plausible si se usa para explicar las cosas de la naturaleza. Por ejemplo, para los científicos es posible sostener que ciertos hechos (como una secuencia particular de ADN) definen la esencia de un tigre, y comprobar, en consecuencia, que el concepto de tigre se usa correctamente si, y solo si, le es atribuido a un animal con el ADN de un tigre. DwORKIN afirma que la idea según la cual nosotros podemos descubrir una estructura ontológica similar en las cosas que no pertenecen a la naturaleza, tal como el derecho, "mediante un proceso totalmente científico, descriptivo, no normativo" es "un sinsentido" 14 . En la misma línea de pensamiento, en Thirty Years On,

11 Para una discusión acerca de esta relación entre los criterios que, por una parte, exponen los fundamentos del derecho, y, por la otra, el significado de la palabra "derecho" y su uso como una objeción al positivismo jurídico, véase Himma, 2002, 155.

12 Acerca de una evaluación del argumento del desacuerdo teórico y una respuesta al mismo véase SHAPIRO, 2007, $50 \mathrm{~s}$.

13 Una pregunta que no es atendida por DwORKIN y que permanece abierta es la referida a si puede existir una explicación diferente de la naturaleza del derecho, que, a la vez, sea el resultado de una generalización empírica y sea resistente a contraejemplos.

14 Dworkin, 2006, 152. 
DwORKIN aduce que la afirmación según la cual el derecho tiene una estructura ontológica que puede ser expuesta únicamente mediante descripciones es una "idea misteriosa". Solo las clases naturales tienen una esencia susceptible de ser descrita:

Los átomos y el DNA de los animales tienen estructuras físicas inherentes, y es lógico suponer que estas estructuras ordenan la "esencia" del hidrógeno o de un león. Pero esto no es comparable de ninguna manera con la naturaleza de una práctica social compleja ${ }^{15}$.

No obstante, Dworkin no provee argumento alguno que soporte esta afirmación escéptica. Tan solo sugiere que en este punto surgiría un dilema y que las dos posibilidades del dilema conducirían a un camino cerrado. Una posibilidad consiste en entender el derecho como un elemento de la naturaleza. Dworkin está en lo correcto cuando rechaza de manera categórica esta posibilidad ${ }^{16}$. La otra posibilidad consiste en pensar que podría existir algo semejante a una estructura ontológica, que sea descriptible, de objetos que, como el derecho, no pertenecen a la naturaleza. Sin embargo, Dworkin afirma que esta aproximación nos conduciría al análisis semántico que él mismo rechaza. La búsqueda de la estructura ontológica del derecho, entendida como una empresa descriptiva, tendría éxito solo en caso de que fuese posible formular una explicación acerca de lo que hace que una característica de los acuerdos sociales implícitos acerca del lenguaje sea esencial para conferirle al derecho su carácter especial, en tanto que hace que otras características sean meramente contingentes. Dworkin piensa que esta explicación solo podría derivar de una reflexión acerca del significado de la palabra "derecho" ${ }^{17}$, y que esta reflexión no conduciría a ningún resultado confiable, debido a la falta de criterios comunes acerca del uso correcto de dicha palabra.

Por otra parte, DwORKIN parece admitir que el derecho tiene una esencia o una estructura ontológica. Este autor sostiene que los conceptos y los valores políticos (según su perspectiva, conceptos tales como libertad, justicia o derecho son conceptos políticos) son "reales"18. Estos conceptos tienen una "estructura profunda" 19 . No obstante, su estructura ontológica es diferente de la estructura de los objetos de la naturaleza. No tienen una estructura "fí-

15 Dworkin, 2002, 1655, reimpreso en Dworkin, 2006, 157-222. Este pasaje se encuentra en 215. De aquí en adelante citaré este artículo a partir de Dworkin, 2006 (Justice in Robes).

16 DwORKIN, 2006, 166: "No necesitamos seguir esta posibilidad [...] porque HART no pudo haber pensado que las afirmaciones verdaderas acerca del derecho se refieren a un elemento de la naturalezza. Si la libertad no tiene ADN, tampoco lo tiene el derecho".

17 DwORKIN, 2006, 153.

18 DWORKIN, 2006, 154.

19 Ibíd. 
sica" sino "normativa". Se trata, en todo caso, de una estructura que puede ser revelada:

igual que un científico puede aspirar [...] a revelar la naturaleza específica de un tigre o del oro mediante la exposición de la estructura física básica de estas entidades, así también un filósofo político puede aspirar a revelar la naturaleza singular de la libertad mediante la exposición de su núcleo normativo ${ }^{20}$.

Sobre la base de sus críticas al análisis conceptual como método para explicar la naturaleza del derecho, DworkIN adopta el procedimiento rawlsiano del equilibrio reflexivo, como una metodología interpretativa alternativa que sería apta para la teoría jurídica ${ }^{21}$.

De los puntos de vista de DwORKIN acerca del proyecto estructural surgen por lo menos dos tipos de preguntas: sustantivas, referidas a si tiene el derecho en realidad una estructura ontológica, y, si es así, a cuál es dicha estructura; y metodológicas, siendo las preguntas centrales a este respecto: ¿cómo puede ser conocida o, como Dworkin sostiene, revelada, dicha estructura ontológica?22, ¿puede ser conocida o revelada por medio de una metodología conceptual?, o, como Dworkin sugiere, ¿puede ser conocida o revelada a través de una metodología interpretativa? ? $^{23}$.

Estos dos tipos de preguntas están íntimamente vinculados. Sin embargo, las preguntas metodológicas son fundamentales con respecto a las preguntas sustantivas. La plausibilidad de toda respuesta a las preguntas sustantivas depende de la plausibilidad de la aproximación metodológica. Esta, a su vez, conduce a toda posible respuesta para las preguntas sustantivas.

En principio parece plausible la intuición según la cual el derecho es real y tiene una estructura ontológica. Los hechos jurídicos, tales como el hecho de que yo esté casado o de que Barack OBama sea el presidente de Estados Unidos, son hechos reales. Ellos tienen un impacto en nuestras vidas, a veces aún más grande que el que tienen los hechos naturales. Además, ellos implican la existencia de ciertos entes jurídicos en el mundo, tales como las cortes o los parlamentos. La existencia de estos entes es innegable. Finalmente, nosotros tenemos de hecho algunas intuiciones incuestionables acerca de la posibilidad de una ontología del derecho. Como Michael Moore ha seña-

20 DwORKIN, 2006, 155.

21 Dworkin, 2003-2004, 1387-1405.

22 Con escepticismo, Patterson presenta esta pregunta de la siguiente manera: "¿cómo puede ser revelada la estructura profunda de los conceptos políticos?", "¿acerca de qué trata la analogía normativa de Dworkin del ADN, del número atómico y de la fórmula molecular?": véase Patterson, 2006, 553.

23 Sin duda hay una tercera posibilidad, que, no obstante, no tendré en cuenta aquí. Esta es la posibilidad de encontrar la estructura ontológica del derecho por medio de una metodología empírica. Sobre esta posibilidad, véase LeITER, 2007a, 180. Véase también LeITER, 2007b. 
lado, la ontología jurídica incluye "entes, tales como normas; propiedades (cualidades, conjuntos, clases), tales como la validez jurídica; y relaciones, tales como las obligaciones jurídicas de una persona a otra" ${ }^{24}$. Sin embargo, no es claro cómo la estructura ontológica puede ser revelada, si por medio de una estrategia conceptual o por medio de una metodología interpretativa.

Este ensayo tiene por objeto proveer una respuesta a esta pregunta metodológica. Afirmaré que la objeción de Dworkin contra el proyecto estructural es injustificada. Mostraré que los resultados de este proyecto, si bien no dilucidan el significado de la palabra "derecho", explican al derecho como el objeto o el ente que constituye el referente de esta palabra ${ }^{25}$. Las teorías comprometidas con este proyecto no son lexicográficas sino ontológicas ${ }^{26}$. Además, esbozaré una metodología que permite explicar la estructura ontológica del derecho. Afirmo que investigaciones de este tipo deben empezar con una comprensión pre-reflexiva del derecho (sección 2); que ellas deben tratar de explicar el derecho mediante una lista de condiciones necesarias y suficientes para la existencia del derecho (sección 3); y que el éxito de esta empresa, si bien depende del análisis conceptual, también necesita de una metodología interpretativa, o, de manera más precisa, de una construcción teórica dirigida a alcanzar un equilibrio reflexivo (sección 4). Esto implica, en contra de DwORKIN, que el equilibrio reflexivo no es una alternativa mejor que el análisis conceptual sino una metodología complementaria.

\section{UNA COMPRENSIÓN PRE-REFLEXIVA DEL DERECHO}

El punto de partida necesario para toda empresa que tenga por objeto descubrir y explicar la naturaleza del derecho es el de seleccionar una comprensión prereflexiva del derecho. El proyecto estructural es una investigación filosófica que debe empezar por la individualización de su objeto: la investigación acerca de lo que llamamos derecho ${ }^{27}$. Para efectos del análisis, debemos primero

24 Moore, 2002, 620.

25 El punto de vista según el cual el objeto de la teoría del derecho es el de explicar el significado de la palabra "derecho" es conocido como el "enfoque lingüístico". Acerca de los problemas de este enfoque véase RAZ, 1996a, 196; RAZ, 2001, 1, F; SHAPIro, 2011, 22.

26 Esto es así, a pesar del hecho de que consideraciones relacionadas con el uso de palabras sin duda jueguen un rol importante en el análisis. Como Austin sostiene, nosotros "indagamos no solo acerca de las palabras... sino también de las realidades a las que nos referimos con las palabras que usamos al hablar. Desarrollamos una conciencia agudizada acerca de las palabras para agudizar nuestra percepción de los fenómenos": Austin, 1956-1957, 8.

27 Esto parece crear circularidad, debido a que el objeto del proyecto estructural es precisamente determinar lo que es el derecho. No obstante, es posible justificar esta circularidad como una clase de circularidad hermenéutica, si entendemos que el proyecto estructural comienza con la individuación de algunos materiales en bruto como un objeto prima facie de la investigación, y continúa, después de una reflexión más profunda, con la creación de una explicación del derecho más refinada. 
asumir un paradigma acerca de lo que es el derecho, y después investigar acerca de las intuiciones comunes asociadas a él. No obstante, se puede acudir a diversas comprensiones pre-teóricas acerca del derecho. De esta forma, la pregunta relevante es: ¿cuál es la comprensión pre-teórica más apropiada para efectos del proyecto estructural?, y ¿cómo debemos identificarla?

La tarea de seleccionar la comprensión pre-teórica más apropiada acerca del derecho debe ser llevada a cabo por medio del análisis conceptual, es decir, un análisis del concepto de derecho ${ }^{28}$.

El análisis conceptual pretende captar la realidad del derecho mediante la comprensión de su concepto. El supuesto básico es que, por una parte, los conceptos median entre el pensamiento y el lenguaje; y por otra, que los conceptos son entes del mundo. Los conceptos son elementos comunes a distintos contenidos del pensamiento que ayudan a determinar aquello de lo que tratan esos pensamientos. Por ejemplo, la creencia de que A ama a B y la creencia de que B ama a A son distintas, pero ambas tienen en común, y ambas usan, el concepto de amor, y en virtud de este representan algo acerca de lo que existe en relación con amar en el mundo. De manera similar, la creencia acerca de que lo que Juan hizo era legal y la creencia acerca de que lo que Juan hizo no era legal comparten el concepto de legalidad. La primera representa la acción de Juan como algo que tiene la propiedad de ser legal; la segunda, representa la acción de Juan como algo que no tiene tal propiedad. Un análisis del concepto de derecho, entonces, tiene por objeto determinar qué propiedades posee el ente que llamamos derecho, y qué clase de entes pueden ser considerados como derecho por medio del entendimiento acerca de cómo el concepto de derecho es usado en las creencias que la gente tiene acerca de él ${ }^{29}$.

Sin embargo, el problema es que el concepto de derecho es, por naturaleza, controversial. Esto significa que no existe una explicación única y común capaz de proveer certeza epistémica acerca del objeto de investigación de la teoría del derecho. No obstante, esto no es equivalente a la falta de existencia de creencias compartidas o compartidas acerca del derecho. De hecho, existen lugares comunes relativos al concepto de derecho. Las diversas explicaciones existentes acerca del derecho enfatizan uno o más de estos tópicos. En nuestro tiempo, uno de dichos tópicos es que el caso paradigmático del derecho es el derecho creado por el Estado ${ }^{30}$. Sobre la base de este paradigma, el ente que llamamos derecho es entendido, entre otras cosas, como una forma de control social; como una estructura de mandatos, normas, reglas o principios; como

28 Acerca de este punto de vista véase Shapiro, 2001, en particular el primer capítulo. Contra este punto de vista: LeITER, 2007a, 131 s. Una respuesta muy perspicaz a LeITER se encuentra en Coleman, 2002, 311-351, y Himma, 2007, 3-36.

29 НiмMA, 2007, 4.

30 HART se refirió a él como el "derecho municipal”, en HART, 1994, 1-17. 
un medio para la institucionalización de la razón, la justicia, la moralidad o la corrección; y como una cierta práctica social normativa ${ }^{31}$. La cuestión aquí es: ¿cuál de estas interpretaciones del derecho es la más apropiada como punto de partida para el proyecto estructural de la teoría del derecho?

Quisiera aducir aquí que la interpretación más apropiada es aquella que considera que la estructura ontológica del derecho se funda en una práctica social, es decir, en la práctica jurídica. Esta afirmación se encuentra soportada en el carácter esencial de la práctica jurídica para la existencia del derecho. Es posible expresar este carácter esencial mediante la siguiente proposición: no puede haber derecho sin práctica jurídica ${ }^{32}$, esto es, no puede haber derecho sin una práctica social que al menos esté compuesta por personas que no solo acepten de manera colectiva algunas reglas como vinculantes en un sentido particular (el sentido que denominamos jurídico), sino que atribuyan a dichas reglas un estatus particular (el estatus de las normas jurídicas); y grupos de agentes (las autoridades oficiales) que no solo creen y apliquen de manera colectiva tales reglas, sino que juzguen casos conforme a los estándares que estas reglas establecen ${ }^{33}$.

Además, el concepto de derecho como práctica jurídica es esencial para la comprensión pre-reflexiva del derecho, antes mencionada. El control social se logra por medio de la práctica jurídica. Mandatos, normas, reglas, principios u otra clase de directivas autoritativas son producidas en la práctica jurídica ${ }^{34}$. Por último, la afirmación según la cual el derecho institucionaliza o debe institucionalizar la razón, la justicia, la moralidad o la corrección solo tiene sentido si se interpreta como una afirmación acerca de algo que, de hecho, ya es propio de la práctica jurídica o si se entiende como una afirmación acerca de lo que debe ser propio de dicha práctica.

El concepto de práctica jurídica es esencial también para las explicaciones acerca de la naturaleza del derecho que han sido presentadas por las dos principales teorías acerca de la naturaleza del derecho: el positivismo y el no positivismo jurídico. Por una parte, algunas de las más emblemáticas

31 Acerca de estas diversas explicaciones de la naturaleza del derecho véase, entre otros, CAIRns, 1949, 556 y, más recientemente, TAMANAHA, 2009, 17-23.

32 Esta es una condición necesaria, es decir, es necesario que exista la práctica jurídica para que exista el derecho. No obstante, dos preguntas permanecen abiertas: la primera, si esta es también una condición suficiente, esto es, si la existencia de la práctica jurídica es suficiente para que exista derecho; y la segunda, cuál es la relación metafísica entre el derecho y la práctica jurídica. Esta relación puede ser de reducción, superveniencia, implicación o dependencia.

33 Uno de los retos de una explicación satisfactoria acerca de la naturaleza del derecho, como resultado del proyecto estructural, es cómo explicar los conceptos de fuerza jurídica vinculante, norma jurídica y funcionarios de la ley por medio de un análisis reductivo, en el sentido de un análisis que no use el adjetivo "jurídico" para explicar la existencia de la práctica jurídica, como una propiedad esencial del derecho. La manera en que es posible ofrecer tal análisis es un asunto que, por el momento, debe dejarse abierto.

34 Sobre este punto véase HART, 1994, 46-48. 
explicaciones del positivismo jurídico están basadas en la creencia según la cual los hechos sociales que crean la posibilidad del derecho estructuran a su vez una práctica o una institución social. Para el efecto citaré solo algunos ejemplos. En el pasaje del epílogo a The Concept of Law, que fue mencionado al inicio de este ensayo, HART afirmó que el objeto de su teoría era el "dar una descripción explicativa y esclarecedora del derecho como una compleja institución social y política, que presenta un aspecto gobernado por normas (y en este sentido, es una institución "normativa')" ${ }^{35}$. En la misma dirección, RAZ sostiene que, "[en] su forma más fundamental, la filosofía jurídica es una investigación acerca de la naturaleza del derecho y de las características fundamentales de las instituciones, así como de las prácticas jurídicas" 36 . Por último, Coleman sostiene que "el objetivo de la teoría del derecho es el de arrojar luces sobre la práctica jurídica real" ${ }^{37}$. Por otra parte, los autores no positivistas generalmente aceptan que el derecho tiene una dimensión fáctica o institucional ${ }^{38}$. La dimensión institucional implica la existencia de una práctica social. No obstante, de manera contraria al positivismo jurídico, los autores no positivistas piensan que esta dimensión institucional no agota la naturaleza del derecho, debido a que la misma está conectada de manera conceptual con la dimensión ideal o crítica de la justicia, la moralidad o la corrección $^{39}$.

Este papel central también se muestra de manera clara incluso en Law's Empire, obra en la cual Dworkin reconoce que el derecho es un "fenómeno social" ${ }^{40}$, y señala que las "teorías generales del derecho [...] tienen por objeto la interpretación del objeto principal y la estructura de la práctica jurídica" ${ }^{41}$; y asimismo en un pasaje de Justice in Robes, en el cual DwORKIN explica que conceptos interpretativos - tales como, desde su perspectiva, el concepto de derecho- "nos alientan a cuestionar y reflexionar acerca de lo que exigen algunas prácticas que hemos construido" 42 .

Estas razones conducen a la conclusión según la cual la comprensión preteórica del derecho más apropiada para el propósito del proyecto estructural de la teoría del derecho es aquella que considera al derecho como un ente fundado en una práctica social. Si esta conclusión es verdadera, entonces el elemento central de una teoría del derecho elaborada desde el punto de vista del proyecto estructural debe ser la explicación de la naturaleza de la

36 Véase RAZ, 2001, 3.

37 Coleman, 2001, 106.

38 Véase Alexy, 2010, 173.

39 Véase Alexy, 2002, $28 \mathrm{f}$.

40 Dworkin, 1986, 13.

41 DWORKIN, 1986, 90.

42 Dworkin, 2006, 10. 
práctica jurídica. De esta forma, surge la pregunta: ¿cuál es la estrategia más apropiada para explicar la naturaleza de la práctica jurídica?

\section{CONDICIONES NECESARIAS Y SUFICIENTES}

La forma más clara de explicar la naturaleza del derecho, en términos del proyecto estructural, es mediante la identificación de las condiciones necesarias y suficientes que un ente debe cumplir con el objeto de fundarse en una práctica jurídica. RAZ y ALEXY están en lo cierto cuando sostienen que la elucidación de la naturaleza del derecho implica enunciar un conjunto de "proposiciones acerca del derecho que sean necesariamente verdaderas" ${ }^{43} \mathrm{o}$ afirmar, "de manera necesaria, verdades acerca del derecho" ${ }^{44}$. Algo es necesariamente verdadero acerca del derecho si, y solo si, es verdad en todos los mundos posibles, es decir, siempre que exista y pueda existir derecho. El referente de estas proposiciones no puede ser "todas las propiedades de la práctica jurídica". La variedad de las normas y las prácticas jurídicas hace que se deba descartar esta posibilidad. Existen hechos sociales singulares que determinan propiedades singulares de cada práctica jurídica en este país o en aquella comunidad como, por ejemplo, el hecho de que la ley islámica esté basada en la Sharia (la expresión concreta de la guía de Dios para la humanidad), o que el derecho consuetudinario de los aborígenes de Australia esté influenciado por sus creencias, conforme a las cuales la tierra y sus criaturas fueron creadas por las acciones de seres ancestrales totémicos en el "tiempo del sueño", por un conjunto de eventos que ocurrió en el pasado y que no solo puede hacerse presente a través de la realización de rituales, sino que además es la fuente de las reglas que gobiernan las relaciones sociales. Como explica RAz, hechos sociales locales como estos "no determinan la naturaleza del derecho, ellos sólo afectan su materialización en prácticas concretas" ${ }^{\prime 4}$. Si esto es verdad, entonces las proposiciones que describen aquellas propiedades de las prácticas jurídicas son meramente contingentes y no verdaderas de manera necesaria. El hecho de que una práctica jurídica islámica o una aborigen tenga una de las propiedades mencionadas se debe tan solo a un asunto contingente, es decir, se debe, en los ejemplos mencionados, a hechos sociales que ocurren en momentos y lugares particulares, como son, de manera respectiva, la Sharia y la aceptación del "tiempo del sueño" como fuentes del derecho. Por esta razón, estas propiedades no pueden ser asumidas como objetos de verdades necesarias acerca del derecho ${ }^{46}$.

43 RAz, 2004, 324-325. Véase también Dickson, 2001, 17.

44 AleXy, 2008, 284.

45 RAz, 1996b, 2.

46 Sobre la distinción entre verdades necesarias y contingentes acerca del derecho véase Dickson, 2001, 18. 
Si la elucidación de la naturaleza de la práctica jurídica implica enunciar un conjunto de proposiciones necesariamente verdaderas, entonces estas proposiciones deben tratar acerca de las propiedades que las prácticas jurídicas presentan de manera necesaria, es decir, propiedades sin las cuales una práctica no sería una práctica jurídica sino un ente distinto a esta ${ }^{47}$. Estas son las propiedades que confieren a la práctica su esencia, su "dentidad"48, o, en otras palabras, las propiedades "esenciales" de la práctica jurídica ${ }^{49}$. En consecuencia, explicar la naturaleza de la práctica jurídica en términos del proyecto estructural es algo que puede ser entendido también como la enunciación de las propiedades esenciales de esta práctica ${ }^{50}$.

Una estrategia metodológica adecuada para explicar las propiedades esenciales de la práctica jurídica es expresar dichas propiedades por medio de una elucidación de las condiciones necesarias y suficientes que debe cumplir un ente para ser una práctica jurídica o, en otras palabras, las condiciones necesarias y suficientes que no pueden faltar en un ente para que este sea una práctica jurídica. Con respecto a ciertos entes en el mundo, por ejemplo, algunos objetos de la naturaleza, es posible distinguir entre su esencia nominal y su esencia real. Esta distinción se remonta a Locke, quien, como es conocido, distinguió entre la esencia nominal y la esencia real de un ente ${ }^{51}$. Mientras la esencia nominal de un ente consiste en las condiciones en las cuales puede ser correctamente usada la palabra que nosotros asociamos con el ente, la esencia real es la constitución atómica del ente, que es la base causal de todas las propiedades observables de la cosa. Se cree que en el caso de los objetos de la naturaleza, tales como el oro, dichas esencias son ampliamente divergentes. Una explicación de la esencia nominal del oro podría ser algo como denso, suave, brillante, de color amarillo, maleable, dúctil, puro y metálico; mientras que una explicación de su esencia real sería algo como el elemento con el número atómico 79. No obstante, en relación con las instituciones sociales, tales como la práctica jurídica, la esencia nominal y la esencia real coinciden. Ellas tienen el mismo referente, y este referente no puede ser explicado de otra forma, de manera exhaustiva, sino en términos de las condiciones necesarias y suficientes en las cuales el concepto de una institución social puede ser usado para referirse a un ente. En relación con la práctica jurídica, estas condiciones necesarias y suficientes expresan, por una parte, el concepto de práctica jurídica -en el sentido de que ellas establecen

47 RAZ, 2004, 324.

48 ShAPIRO, 2011, 10.

49 Véase RAz, 2004, 324.

50 Véase, por ejemplo, Coleman, 2001, 108: “[La teoría del derecho] comienza por preguntarse si existen características del derecho que son esenciales o, en un sentido apropiado, necesarias al derecho o a nuestro concepto acerca de él: son esenciales a nuestro concepto en el sentido de que una práctica social que no las tiene no puede ser calificada como derecho".

51 Véase LocKe, 1989, lib. III, cap. III. 
cuándo es apropiado usar este concepto respecto de un ente-, y por la otra, las propiedades esenciales del ente que llamamos práctica jurídica, es decir, la naturaleza de la práctica jurídica. De esta forma, dichas condiciones necesarias y suficientes crean una correlación entre el concepto y la naturaleza de la práctica jurídica, de tal modo que las condiciones necesarias y suficientes que un ente debe tener para ser una práctica jurídica también establecen las condiciones en las cuales el concepto de práctica jurídica puede ser usado con respecto a ella de manera adecuada. Debido a esta correlación, una elucidación de las condiciones necesarias y suficientes que un ente debe tener para ser una práctica jurídica es, al mismo tiempo, tanto una explicación metafísica de la naturaleza del derecho como una explicación del concepto de derecho en tanto entidad fundada en una práctica jurídica.

En este punto surgen dos preguntas: la primera se refiere a si es posible afirmar un conjunto de condiciones necesarias y suficientes que un ente debe cumplir para ser una práctica jurídica; la segunda se refiere a si esta estrategia nos proporciona la mejor explicación posible acerca de la naturaleza de dicha práctica.

Respecto a la primera pregunta, no puede ser descartada de plano la posibilidad de admitir un análisis reductivo del concepto de derecho, en tanto entidad fundada en una práctica jurídica, en el sentido de un análisis que provea una explicación acerca de las condiciones de uso de dicho concepto en términos de conceptos más básicos. Algunos conceptos permiten un análisis reductivo puro, en términos de un conjunto de condiciones necesarias y suficientes. Un ejemplo de este tipo de conceptos es el concepto de círculo: para todo $\mathrm{x}, \mathrm{x}$ es un círculo si, $\mathrm{y}$ solo si, $\mathrm{x}$ es un conjunto de puntos equidistantes de un punto dado en el plano ${ }^{52}$. Este análisis del concepto de círculo provee una descripción adecuada de la naturaleza de un círculo. El problema con los análisis puros, como este, radica en el hecho de que es menos probable que sea adecuado si lo usamos con la finalidad de intentar una explicación de conceptos (más complejos que el concepto de círculo) que se usan para hacer referencia a un grupo heterogéneo de cosas, que si lo usamos con el objeto de intentar una explicación de conceptos que no están respaldados por un consenso acerca de sus condiciones de uso. La pregunta es, entonces, si el concepto de práctica jurídica admite un análisis como este.

Esta posibilidad no puede, sin embargo, descartarse, salvo que se demuestre lo contrario. Debido a que la explicación de la naturaleza de un ente por medio de la afirmación de las condiciones necesarias y suficientes del concepto relacionado con dicho ente ofrece el grado máximo de claridad, la investigación de la teoría del derecho acerca de la naturaleza de la práctica

Doy las gracias a KIRK LUDWIG por sus esclarecedores comentarios acerca de este punto. 
jurídica debe tener por objeto la identificación de dichas condiciones, con el objeto de lograr tal grado de claridad.

Un segundo asunto radica, ya no en el hecho de si es posible explicar la naturaleza de la práctica jurídica por medio de un conjunto de condiciones necesarias y suficientes, sino en si esta estrategia es la más apropiada para este propósito. Raz reconoce que, "con más frecuencia de lo que a veces se supone", las explicaciones acerca de la naturaleza de cosas, tales como el derecho, pueden elaborarse mediante explicaciones que ofrecen "condiciones necesarias y suficientes para el uso" del concepto ${ }^{53}$. RAZ nos advierte, sin embargo, que "es un error creer que toda buena explicación debe" ser elaborada de esta forma ${ }^{54}$. Aún más, este autor parece incluso considerar que las explicaciones en términos de condiciones necesarias y suficientes pueden implicar enormes desventajas. De manera particular, considera que este tipo de explicaciones pueden dificultar el logro de la finalidad de explicar la naturaleza de un ente, debido a que pasan por alto la "importancia de otras características" ${ }^{55} \mathrm{o}$ las conexiones entre el concepto en cuestión y otros $\operatorname{conceptos}^{56}$, y porque ellas pueden conducir a una falsa imagen acerca de lo que las explicaciones buscan lograr -es decir, la falsa imagen según la cual solo una lista completa de "condiciones necesarias y suficientes proporciona una explicación exhaustiva" de la naturaleza de una cosa- ${ }^{57}$.

La advertencia de RAZ no puede ser interpretada, sin embargo, como una evidencia dirigida a probar que la explicación de la naturaleza de un ente, como la práctica jurídica, por medio de un conjunto de condiciones necesarias y suficientes, no es la más apropiada. Consideraré los argumentos de Raz de manera retrospectiva. En primer lugar, dicha estrategia no es naturalmente la única estrategia posible para explicar la naturaleza de un ente. No obstante, ella tiene la ventaja de ofrecer el mayor grado de claridad. La claridad es un ideal que toda explicación teórica debe buscar. En segundo lugar, la explicación acerca de la naturaleza de un ente $\phi$, por medio de un conjunto de condiciones necesarias y suficientes, no implica pretermitir la conexión del concepto de $\phi$ con otros conceptos. La explicación misma acerca del concepto y la naturaleza de $\phi$, por medio de un conjunto de condiciones necesarias y suficientes, siempre que sea una explicación reductiva en el sentido anteriormente indicado, es decir, una explicación en términos de conceptos más básicos, implica el hecho de que subyacen conexiones entre el concepto de $\phi$ y otros conceptos. Un concepto no permitiría una explicación en términos de condiciones necesarias y suficientes si fuese el concepto fundamental; esto

\footnotetext{
53 RAZ, 2001,9.

54 Ibíd.

55 RAZ, 2001,9.

56 RAz, 2001, 10.

57 Ibíd.
} 
es, si este concepto no admitiese una explicación reductiva en términos de conceptos más básicos. En este caso, la única estrategia aceptable radica en explicar este concepto básico por medio de la explicación de sus conexiones con otros conceptos básicos. No obstante, este no es el caso de la práctica jurídica, que no es un concepto fundamental en este sentido. Una práctica es un conjunto de acciones llevadas a cabo por algunos agentes relevantes. Así, acciones y agentes son conceptos fundamentales respecto de una práctica jurídica. Finalmente, RAZ no afirma que otras propiedades adicionales a aquellas esenciales deban ser incluidas en una explicación acerca de la naturaleza de un ente, sino que la importancia de otras propiedades puede ser pasada por alto, con base en una explicación que tenga la forma de un conjunto de condiciones necesarias y suficientes. Sin duda, este es un riesgo cierto. Sin embargo, este riesgo es más bien bajo, dado que la tarea misma de elaborar una lista de condiciones necesarias y suficientes implica decidir acerca de cuáles propiedades son esenciales para la naturaleza de un ente, cuáles son meramente importantes, y cuáles no son ni esenciales ni importantes. La deliberación acerca del carácter esencial de las propiedades de un ente garantiza que toda propiedad importante sea tomada en cuenta, a pesar del hecho de que, al final, el teórico del derecho tenga buenas razones para incluir o excluir algunas de ellas del conjunto definitivo de condiciones necesarias y suficientes. Esta deliberación puede ser realizada por medio de un equilibrio reflexivo que, a su vez, puede satisfacer el propósito de las explicaciones teóricas. Dicho propósito, de acuerdo con RAZ, radica en alcanzar "un entendimiento más sistemático acerca de los conceptos", es decir, un entendimiento "que no sólo guiará el uso correcto [de los conceptos], sino que mejorará su comprensión" 58 .

En cuanto a este último punto, en un artículo reciente, SCHAUER sostiene que una explicación apropiada acerca de la naturaleza del derecho debe no solo incluir las propiedades necesarias y suficientes, sino también "aquellas propiedades que sean importantes, aunque no necesarias" ${ }^{59}$. Las propiedades "importantes" de un ente comprenden, según este, aquellas propiedades que no son exclusivas del derecho "pero que están, en un sentido empírico y probabilístico, concentradas en" él. SCHAUER menciona, como ejemplos de propiedades importantes, el "volar" en el caso de las aves o la "coerción" en el caso del derecho. A pesar del hecho de que no todas las aves vuelan y que no todo el derecho es coercitivo, el volar y la coerción son propiedades importantes de estos entes, debido a que ellas se encuentran empíricamente concentradas en tales entes y su inclusión en la explicación de la naturaleza de estos enriquece nuestro entendimiento tanto respecto de las aves como del derecho. No obstante, no es claro $-\mathrm{y}$, aun si Schauer estuviese en lo

59 SCHAUER, 2010. 
cierto, él no ofrece explicación alguna acerca de ello-, por una parte, qué grado de concentración probabilística es suficiente, necesaria o importante para que una propiedad sea una propiedad importante de un ente en general, y del derecho, en particular; y por otra, cómo deben emplearse los métodos probabilísticos para permitirnos identificar las propiedades importantes del derecho y para distinguirlas de aquellas propiedades no importantes.

Finalmente, de acuerdo a una perspectiva más amplia que se remonta a las Philosophical Investigations de WitTGenstein ${ }^{60}$, pueden existir también conceptos que se aplican a cosas mediante la metodología del parecido de familia (en alemán: Familienähnlichkeit) que tienen con un ejemplo paradigmático ${ }^{61}$. La idea de WitTGENSTEIN es que los entes no caen bajo un concepto que se considera que cumple con un conjunto de condiciones necesarias y suficientes; por el contrario, los entes están conectados por una serie de similitudes coincidentes y no tienen una propiedad común a todos. En este sentido, en el análisis de un concepto (como el concepto de juego, usado por WitTGENSTEIN) nosotros debemos relacionar los diversos usos de una palabra mediante una "complicada red de parecidos que se superponen y entrecruzan" ${ }^{2}$. En tanto que no existen límites en relación con los diferentes usos de un mismo concepto, no existen condiciones necesarias y suficientes que todos los entes compartan ${ }^{63}$, cuando caen bajo el mismo concepto.

Puede ocurrir que, después de tratar de encontrar un conjunto de condiciones en el cual cada una de ellas sea necesaria y que, en conjunto, sean suficientes para el concepto de la práctica jurídica, el resultado de la investigación de la teoría del derecho sea precisamente que no existen tales condiciones, capaces de explicar la naturaleza de la práctica jurídica y de diferenciarla de otros entes. Puede ocurrir también que el concepto de práctica jurídica sea usado en relación con entes diversos que se relacionan meramente por medio de una red de similaridades que se superponen y entrecruzan. Además, puede ocurrir que el concepto de práctica jurídica sea una categoría en la cual exista un concepto prototipo de práctica jurídica, como el concepto de silla dentro del ámbito del concepto de mobiliario, de manera tal que los entes que caigan en el concepto de práctica jurídica puedan ser solo entendidos como una variación de un prototipo ${ }^{64}$. No obstante, por una parte, la mejor manera de llegar a concluir que la práctica jurídica es un concepto que debe analizarse con el método del parecido de familia es mostrando que no es posible identificar un conjunto de condiciones necesarias y suficientes que

60 Wittgenstein, 2001.

61 Acerca de las condiciones necesarias y suficientes y de su aplicación, y acerca del parecido de familia como una forma alternativa de expresar un concepto, véase Strawson, $1959,11$.

62 WitTGENSTEIN, 2001, § 65-71.

63 Anat Biletzki y Matar Anat, 2009.

64 Rosch, 1973, 328-350. 
un ente debe tener para ser una práctica jurídica; y por otra, la mejor manera de explicar un concepto prototipo de una práctica jurídica es por medio de la enunciación de un conjunto de condiciones que expliquen la naturaleza de este concepto prototipo. Por estas razones, identificar este conjunto de condiciones necesarias y suficientes debe ser todavía el objetivo de la investigación de la teoría del derecho.

\section{EQUILIBRIO REFLEXIVO}

Solo queda por responder la pregunta final, a saber, cómo establecer el conjunto de condiciones necesarias y suficientes de la práctica jurídica. En este punto aduciré que el equilibrio reflexivo es un método apropiado para llevar a cabo esta tarea.

No existe algo así como un algoritmo para llevar a cabo una investigación filosófico-jurídica. Dworkin está en lo cierto cuando sostiene que la creación de una explicación acerca de la naturaleza del derecho exige algo más que una descripción. En cierto modo, esto es bastante obvio en relación con toda teoría que necesite una reflexión crítica. En tanto teoría, una explicación acerca de la naturaleza de la práctica jurídica se basa en la existencia de ciertas creencias, y en particular, de ciertas intuiciones. Las intuiciones son creencias acerca de la verdad de una proposición; verdad que, al mismo tiempo, como Depaul afirma, el investigador "en realidad no adquiere por medio de la percepción, la introspección, la memoria, la evidencia o porque la haya inferido de manera explícita de la proposición", sino simplemente porque "la proposición parece [a dicha persona] verdadera una vez la ha examinado debidamente" 65 .

Cuando un investigador usa el análisis conceptual para investigar la naturaleza del derecho, las teorías resultantes de dicho análisis descansan en intuiciones y en otro tipo de creencias acerca de la naturaleza del derecho. En cierto sentido, es claro que esto no puede ser suficiente. Muchas de estas intuiciones podrían llegar a ser incompatibles entre sí. Esto conduce no solo a que dichas intuiciones deben ser asumidas como revisables, sino a que la investigación teórico jurídica debe comprender también un proceso que permita valorar las intuiciones conflictivas en el amplio ámbito referente a la explicación de la práctica jurídica. De esta forma, el investigador debe emprender un proceso de reflexión, con el fin de decidir acerca de cuáles intuiciones y creencias deben permanecer, cuáles deben ser revisadas y cuáles descartadas ${ }^{66}$. Este proceso es conocido como equilibrio reflexivo.

66 Ross, 1930, 40-41. 
El concepto de equilibrio reflexivo fue introducido en el ámbito de la filosofía por Nelson GoOdMAN ${ }^{67}$, como un proceso de ajuste mutuo de creencias referentes a la justificación de reglas de la lógica inductiva y deductiva. Según su idea inicial, debe existir coherencia entre nuestras creencias respecto de las reglas de la lógica inductiva y deductiva, de forma tal que una regla no sería aceptable como principio lógico si ella no es compatible con lo que nosotros asumimos como ejemplos aceptables de razonamiento inferencial. Con el objeto de evaluar esta aceptabilidad, debemos emprender un proceso de corrección y revisión de nuestros puntos de vista acerca de todas nuestras creencias referentes a estas reglas.

En el ámbito de la ética y de la filosofía política, el equilibrio reflexivo fue explicado y desarrollado por JoHn Rawls en A Theory of Justice, como un método dirigido a examinar nuestros juicios morales acerca de un asunto particular mediante la búsqueda de su coherencia con nuestras creencias respecto de casos similares y nuestras creencias respecto de asuntos morales, y al mismo tiempo, como resultado del proceso en el que se lleva a cabo este examen. En este segundo sentido, RaWLS afirmó que el equilibrio reflexivo debe ser el resultado del proceso que usamos para encontrar los principios que expresan los términos de cooperación justa que deben gobernar una sociedad compuesta por agentes libres y moralmente iguales. Este proceso debe empezar con la afirmación de nuestras "convicciones meditadas acerca de la justicia" 68 . Estas convicciones son "puntos fijos provisionales que suponemos debe satisfacer cualquier concepción de la justicia" ${ }^{69}$. Examinamos a continuación si los posibles principios que expresan los términos de una cooperación justa coinciden con nuestras convicciones meditadas acerca de la justicia. En caso de discrepancia, podemos elegir o rechazar todos los principios en juego, o revisar algunas de nuestras convicciones meditadas de la justicia. Una vez hemos logrado la consistencia entre los principios en juego y las convicciones meditadas acerca de la justicia, alcanzamos el equilibrio reflexivo ${ }^{70}$. No obstante, aun este equilibrio es provisional. Está siempre abierto y es revisable, siempre y cuando surjan nuevas consideraciones relevantes.

Hoy en día, el equilibrio reflexivo es considerado como "el punto final de un proceso deliberativo mediante el cual reflexionamos y revisamos nuestras creencias acerca de un área de investigación, sea ésta moral o no moral"71.

Como Daniels explica, este método consiste en "moverse una y otra vez a través de nuestros juicios meditados" (o intuiciones) revisándolos cada

68 Rawls, 1971, 19.

69 Ibíd.

70 Ibíd.

71 DANIELS, 2003. 
vez que sea necesario "con el objeto de lograr una coherencia constante entre" ellos ${ }^{72}$. El método funciona si, por medio de él, logramos llegar a un equilibrio reflexivo, esto es, "si llegamos a una coherencia aceptable entre nuestras intuiciones"73. Coherencia aceptable significa que nuestras intuiciones no se contradicen entre sí y proveen fundamentos que ayudan a explicar el sistema del cual forman parte. Esta forma de coherencia se logra cuando "no estamos inclinados a revisarlas [nuestras intuiciones] de nuevo, debido a que ellas, como un todo, tienen el más alto grado de aceptabilidad o credibilidad para nosotros" 74 .

La distinción entre equilibrio reflexivo amplio y restringido es bien conocida en el campo de la ética y de la filosofía política. El equilibrio reflexivo restringido es un método de deliberación moral en el cual un investigador moral indaga, alternando entre un juicio acerca de un asunto moral y las razones que justifican dicho juicio ${ }^{75}$. Este proceso comienza con las intuiciones del investigador moral acerca de la posible solución a un problema moral. Una reflexión más profunda respecto de estas intuiciones conduce a la expresión, por parte del investigador, de sus juicios morales considerados acerca del asunto en cuestión. Sobre esta base, el investigador formula una teoría moral que explica sus juicios morales considerados, los cuales justifica por medio de ciertos principios. Al comienzo del proceso, como sugiere RAwLs, el investigador sostiene sus juicios meditados o considerados de manera inalterada, mientras formula los principios relevantes; una vez formulados, el investigador somete a prueba dichos principios en relación con sus juicios meditados y, o bien reformula sus principios de manera concordante con los juicios, o bien revisa estos últimos. Por último, el investigador toma en cuenta los posibles conflictos entre sus intuiciones, sus juicios morales meditados o considerados, y los principios morales, explicándolos y justificándolos, afirmando así una teoría revisada. El proceso total implica un movimiento oscilante entre los juicios intuitivos y los principios generales. El resultado del proceso es una teoría estable y coherente que no necesita revisiones ulteriores.

El equilibrio reflexivo amplio es un paso más en la investigación moral. Es un proceso en el que el investigador considera teorías alternativas a su teoría moral, y evalúa los argumentos que pueden ser usados a favor y en contra no solo de teorías alternativas, sino de su propia teoría ${ }^{76}$. El equilibrio reflexivo amplio implica tanto una comparación entre la teoría propia y teorías alternativas, en respuesta a los posibles retos a las intuiciones propias originales, juicios meditados y principios, como la comparación de las ventajas

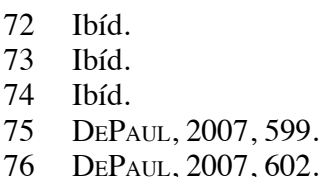


y desventajas de la teoría propia con las ventajas y desventajas de teorías alternativas ${ }^{77}$. El equilibrio reflexivo amplio puede implicar también teorías empíricas y otras teorías fundamentales en la comparación.

Una teoría moral y una teoría acerca de la naturaleza de un ente comparten algunos elementos. Ambas tienen por objeto facilitar la comprensión de manera respectiva, o bien de lo que es la solución correcta a un problema moral, o bien de lo que son las propiedades esenciales de un ente. Además, ambas están compuestas por intuiciones y otro tipo de creencias, juicios meditados y principios o afirmaciones explicatorias y justificatorias. Por esta razón, parece posible usar el equilibrio reflexivo, mutatis mutandi, también como un método para construir una teoría acerca de la naturaleza de un ente como el derecho.

La pregunta final es: ¿cómo usar el equilibrio reflexivo en este paso metodológico final para la construcción de una explicación de la estructura ontológica del derecho?

De la explicación anterior es posible concluir que dos pasos son necesarios: primero, la construcción de un equilibrio reflexivo restringido, y a continuación, la construcción de un equilibrio reflexivo amplio.

A los efectos de la construcción del equilibrio reflexivo restringido debemos empezar con intuiciones muy básicas y otros tipos de creencias acerca de la práctica jurídica, tales como las creencias relacionadas con las siguientes afirmaciones: (1) La práctica jurídica está compuesta por acciones, y en particular, por acciones intencionales. Dado que las acciones son llevadas a cabo por agentes, entonces, (2) existen agentes que participan en la práctica jurídica. (3) Estos agentes deben tener alguna clase de actitud subjetiva respecto de la práctica jurídica. (4) Estos agentes llevan a cabo la práctica jurídica de manera colectiva. Esto significa que la práctica jurídica no es el resultado de acciones de uno o más individuos aislados, ni la acción de un súper-agente, sino de acciones colectivas intencionales de agentes relevantes. (5) La práctica jurídica tiene por objeto regular el comportamiento. Entonces, (6) los agentes, de manera colectiva, crean, aplican, juzgan, cambian, cumplen con estándares normativos de comportamiento.

A partir de estas creencias, es posible construir una explicación coherente y detallada no solo acerca de lo que son las acciones intencionales dentro de este contexto y también acerca de cuáles son los agentes relevantes de la práctica jurídica, sino incluso acerca de lo que son sus actitudes subjetivas relevantes y acerca de cómo se debe entender el actuar y el vivir dentro de una práctica jurídica, y especialmente, acerca de cómo es posible para los agentes relevantes crear, aplicar, juzgar, cambiar y cumplir estándares normativos de comportamiento. Después de la construcción de esta explicación, es 
necesario evaluarla en relación con las creencias originales afirmadas arriba. El resultado será una teoría revisada de la práctica jurídica.

El objetivo de este segundo paso es el de lograr un equilibrio reflexivo amplio. Este segundo paso implica una comparación entre la explicación propuesta de la estructura ontológica del derecho y otras teorías sobre la naturaleza del mismo. La construcción de una explicación de la estructura ontológica del derecho que sea capaz de lograr esta clase de equilibrio reflexivo restringido es objeto de una investigación más amplia. Este ensayo es tan solo el primer paso.

\section{BIBLIOGRAFÍA}

Alexy, R. (2002). The Argument from Injustice. A Reply to Legal Positivism, Oxford, Oxford University Press; en castellano, ALEXY, R. El concepto y la validez del derecho,

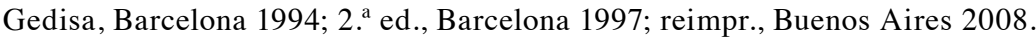

Alexy, R. (2008). On the Concept and the Nature of Law, en Ratio Juris, 21, 3, 281-299; en castellano, Alexy, R. El concepto y la naturaleza del derecho, Marcial Pons, Barcelona, 2008.

Alexy, R. (2010). The Dual Nature of Law, en Ratio Juris, 23, 2, 167-182; en castellano, Alexy, R. La doble naturaleza del Derecho, en Carlos Bernal Pulido (ed.), La doble dimensión del Derecho. Autoridad y razón en la obra de RoBERT Alexy, Palestra, Lima, 2011, 29-58.

Austin, J. (1956-1957). A Plea for Excuses, en Proceedings of the Aristotelian Society, 57, 1-30; en castellano, Austin, J. Un alegato en pro de las excusas, en Austin, J., Ensayos filosóficos, Revista de Occidente, Madrid, 1975, 169-192.

Biletzki, A. y Matar, A. (2009). Wittgenstein, en Stanford Encyclopedia of Philosophy, en: http://plato.stanford.edu/entries/wittgenstein/ (05.07.2012).

Cairns, H. (1949). Legal Philosophy from Plato to Hegel, Baltimore, Johns Hopkins Press.

Coleman, J. (2001). Incorporationism, Conventionality, and the Practical Difference Thesis, en Id. (ed.), HaRT's Postscript. Essays on the Postscript to the Concept of Law, Oxford, Oxford University Press.

Coleman, J. (2002). Methodology, en Id. y Shapiro, S. (eds.), The Oxford Handbook of Jurisprudence and Philosophy of Law, Oxford, Oxford University Press, 311-351.

Daniels, N. (2003). Reflective Equilibrium, en Stanford Encyclopedia of Philosophy, en: http://plato.stanford.edu/entries/ reflective-equilibrium/ (05.07.2012).

DePaul, M. (2007). Intuitions in Moral Inquiry, en Copp, D. (ed.), Oxford Handbook of Ethical Theory, Oxford, Oxford University Press.

Dickson, J. (2001). Evaluation and Legal Theory, Oxford, Hart Publishing.

Dworkin, R. (1986). Law's Empire, Cambridge Mass., Harvard University Press; en castellano, Dworkin, R. El imperio de la justicia, Gedisa, Barcelona, 1988. 
Dworkin, R. (2002). Thirty Years On, en Harvard Law Review, 115, 1655-1687.

Dworkin, R. (2003-2004). Rawls and the Law, en Ratio Juris, 72, 1387-1405.

Dworkin, R. (2004). Hart's Postscript and the Character of Political Philosophy, en Oxford Journal of Legal Studies, 24, 1, 1-37; en castellano, Dworkin, R. El post scriptum de Hart y el carácter de la filosofía política, en Orellana Benado, M. (ed.), Causas perdidas: ensayos de filosofía, jurídica, política y moral, Catalonia, Santiago de Chile, 2010, 2-34.

Dworkin, R. (2006). Justice in Robes, Cambridge, Mass., Belknap Press of Harvard University Press; en castellano, Dworkin, R. La justicia con toga, Marcial Pons, Barcelona, 2007.

Dworkin, R. (2011). Justice for Hedgehogs, Cambridge, Mass., Belknap Press of Harvard University Press.

Goodman, N. (1955). Fact, Fiction, and Forecast, Cambridge, Mass., Harvard University Press; en castellano, Goodman, N. Hecho, ficción y pronóstico, Síntesis, Madrid, 2004.

Himma, K. E. (2002). Ambiguously Stung: Dworkin's Semantic Sting Reconfigure, en Legal Theory, 8, 2, 145-184.

Himma, K. E. (2007). Reconsidering a Dogma: Conceptual Analysis, the Naturalistic Turn, and Legal Philosophy, en Freeman, M. y Harrison, R. (eds.), Law and Philosophy. Current Legal Issues, Oxford, Oxford University Press, 3-36.

Hart, H.L.A. (1994). The Concept of Law, Oxford, Clarendon Press. En castellano, Hart, H.L.A. El concepto del Derecho, 2. ${ }^{\text {a }}$ ed., reimpr., Abeledo-Perrot, Buenos Aires, 2007.

LeIter, B. (2007a). Naturalizing Jurisprudence. Essays on American Legal Realism and Naturalism in Legal Philosophy, Oxford, Oxford University Press; en castellano, LeITER, B. Naturalismo y teoría del derecho, Marcial Pons, Barcelona, 2012.

Leiter, B. (2007b). Naturalism in Legal Philosophy, en Stanford Encyclopedia of Philosophy, en: http://plato.stanford.edu/entries/ lawphil-naturalism/ (05.07.2012).

Locke, J. (1989). An Essay Concerning Human Understanding, Oxford, Clarendon Press; en castellano, Locke, J. Ensayo sobre el entendimiento humano, Fondo de Cultura Económica, México, 1999.

Moore, M. (2002). Legal Reality: A Naturalist Approach to Legal Ontology, en Law and Philosophy, 21, 619-705.

Patterson, D. (2006). Dworkin on the Semantics of Legal and Political Concepts, en Oxford Journal of Legal Studies, 26, 3, 553.

Rawls, J. (1971). A Theory of Justice, Cambridge, Mass., The Belknap Press of Harvard University Press; en castellano, Rawls, J. Teoría de la justicia, Fondo de Cultura Económica, México, 1997.

Raz, J. (1996a). The Problem about the Nature of Law, en Id., Ethics and the Public Domain. Essays in the Morality of Law and Politics, Oxford, Clarendon Press, 195-213; en castellano, R Az, J. El problema de la naturaleza del derecho, en Isonomía. Revista de Teoría y Filosofía del Derecho, 3, 1995, p. 131-151. 
RAz, J. (1996b). On the Nature of Law, en Archiv für Rechts- und Sozialphilosophie, 82, 1-25.

Raz, J. (2001). Two Views of the Nature of the Theory of Law. A Partial Comparison, en Coleman, J. (ed.), Hart's Postscript. Essays on the Postscript to the Concept of Law, Oxford, Oxford University Press.

RAz, J. (2005). Can There Be a Theory of Law?, en Golding, M. P. y Edmundson W. A. (eds.), The Blackwell Guide to the Philosophy of Law and Legal Theory, Oxford, Blackwell, 324-342; en castellano, RAz, J. ¿Puede haber una teoría del derecho?, en RAz, J. Una discusión sobre la Teoría del Derecho, Marcial Pons, Barcelona, 2007.

Rosch, E. (1973). Natural categories, en Cognitive Psychology, 4, 328-350.

Ross, W.D. (1930). The Right and Good. Oxford, Oxford University Press; en castellano, Ross, W. D., Lo correcto y lo bueno, Sígueme, Salamanca, 1994.

Schauer, F. (2010). Necessity, Importance, and the Nature of Law, en: http://papers.ssrn. com/sol3/papers.cfm?abstract_id=1594930 (12.09.2011).

Shapiro, S. (2007). The "Hart-Dworkin" Debate. A Short Guide for the Perplexed, en Ripstein, A. (ed.), Ronald Dworkin, Cambridge, Cambridge University Press, 22-55.

Shapiro, S. (2011). Legality, Cambridge, Mass., Harvard University Press.

Sмith, D. (2010). Theoretical Disagreement and the Semantic Sting, en Oxford Journal of Legal Studies, 30, 4, 635-661.

Stavropoulos, N. (2003). Interpretivist theories of Law, en Stanford Encyclopedia of Philosophy, en: http://plato.stanford.edu/entries/law-interpretivist/ (05.07.2012).

Strawson, P.F. (1959). Individuals. An Essay in Descriptive Metaphysics, London, Methuen; en castellano, Strawson, P.F. Individuos: Un ensayo en metafísica descriptiva, Taurus, Madrid, 1989.

Tamanaha, B. (2009). Law, en Katz, S. N., Oxford International Encyclopedia of Legal History, Oxford, Oxford University Press, vol. 4, 17-23.

Wittgenstein, L. (2001). Philosophical Investigations. The German Text, with a Revised English Translation (G.E.M. Anscombe, trans.), London, John Wiley \& Sons; en castellano, Wittgenstein, L. Investigaciones filosóficas, en Wittgenstein, t. I, Gredos, Madrid, 2009, 155-633. 